\title{
Diarrea crónica en el adulto: un reto diagnóstico
}

\author{
Chronic diarrhea in adults: a diagnostic challenge
}

\author{
Albino Pérez Carnero \\ Servicio de Medicina Interna. Hospital Universitario Lucus Augusti. SERGAS. Lugo.
}

La diarrea crónica se define como aquella que tiene una duración superior a 4 semanas ${ }^{1}$ y se caracteriza por una disminución en la consistencia de las heces y/o un aumento en el número de deposiciones.

Es una dolencia frecuente con una prevalencia estimada del $5 \%$ en la población occidental ${ }^{1,2}$. Por el aspecto de las heces la diarrea puede clasificarse como: acuosa, grasa, que guarda relación con maldigestión o malabsorción, y con sangre, que es debida a inflamación y daño en la pared intestinal ${ }^{1,3}$ (tabla 1).

Tabla 1. Clasificación de la diarrea según el aspecto de las heces y su patogenia

\begin{tabular}{ll}
\hline Acuosa & Osmótica \\
& Secretora \\
& Hipermotilidad \\
& Mixta \\
\hline Grasa & Maladigestión \\
& Malabsorción \\
\hline Hemática & Inflamatoria \\
& Infecciosa \\
& Tumoral \\
\hline
\end{tabular}

1. La diarrea acuosa puede producirse por un mecanismo osmótico, secretor o de hipermotilidad.

a. La diarrea osmótica se produce por la ingesta de productos con alto poder osmótico que retienen agua y aumentan el contenido líquido intestinal. En este grupo se incluyen la toma de algunos laxantes y anti-ácidos $(\mathrm{Mg})$, así como la intolerancia a la lactosa, fructosa y sorbitol. La diarrea osmótica cede con el ayuno.

b. En la diarrea secretora hay aumento de la producción intestinal de líquido y electrolitos, bien sea por la acción de enterotoxinas o de sustancias hormonales como las que producen los tumores neuroendocrinos. La diarrea secretora a diferencia de la osmótica no guarda relación con la ingesta y persiste durante las 24 horas del día. c. La diarrea por hipermotilidad es de naturaleza funcional, supone el $50 \%$ de las diarreas crónicas y la mayoría se encuadran en el síndrome de intestino irritable (SII).

2. Las diarreas con alto contenido graso $0^{4}$ (superior a $14 \mathrm{grs}$ dia) pueden ser debidas a maladigestión ó malabsorción. Las heces son espumosas, brillantes y suelen flotar en el agua. La causa más frecuente de maladigestión es la pancreatitis crónica, y las causas de malabsorción, que la mayoría de las veces es expresión de enfermedad del intestino delgado, pueden ser mútiples, con ejemplos como la enfermedad celiaca, el linfoma intestinal, la enfermedad de Whipple etc. Al existir afectación mucosa el diagnóstico se hace casi siempre por biopsia intestinal.

3. Las diarreas inflamatorias se caracterizan por la presencia en las heces de leucocitos y sangre macro ó microscópica, traducen enfermedad orgánica y daño en la pared intestinal. El ejemplo más representativo es la enfermedad inflamatoria crónica intestinal (EICI) (colitis ulcerosa y Crohn) ${ }^{1}$. Dentro de este grupo se incluyen además las infecciones intestinales enteroinvasivas y los tumores.

\section{Aproximación diagnóstica para el estudio de una diarrea crónica en el adulto}

Al igual que en cualquier área de la medicina, la realización de una buena historia clínica es fundamental para orientar el estudio de una diarrea crónica. Los datos que obligatoriamente deben de recogerse son los siguientes:

- Duración de la diarrea, número de deposiciones, periodicidad horaria, volumen y aspecto de las heces.

- Dolor abdominal y sus características.

- Síntomas generales como son, pérdida de peso, fiebre, artralgias etc.

- Factores agravantes, estrés, alimentos lácteos y azúcares.

- Ingesta de fármacos.

- Enfermedades asociadas: diabetes, tiroides, neoplasias, $\mathrm{VIH}$, etc.

- Antecedentes de cirugía previa, radioterapia, y de viajes a otros paises.

- Antecedentes familiares de E.I.C.I, celiaca y neoplasias 


\section{Al igual que en cualquier área de la medicina, la realización de una buena historia clínica es fundamental para orientar el estudio de una diarrea crónica}

En el examen físico, es preciso valorar las alteraciones cutáneas que suelen asociarse a endocrinopatías, la existencia de adenopatías, masas, visceromegalias, meteorismo, artritis, fiebre, sin olvidar la inspección anal, tacto rectal y la valoración del tono esfinteriano (incontinencia fecal). Con todos estos datos podemos hacer un diagnóstico diferencial entre diarrea orgánica o funcional y orientar debidamente las peticiones de pruebas complementarias.

Son datos de organicidad, el dolor abdominal progresivo, la presencia de sangre en heces, el no respetar el sueño, y un síndrome general con adelgazamiento, astenia, artralgias, fiebre, lesiones cutáneas, etc ${ }^{1}$.

La analítica de sangre debe incluir en principio las determinaciones siguientes: hemograma, VSG, bioquímica general, iones, albúmina, ferrocinética y función tiroidea ${ }^{1,2}$. En ocasiones es necesario determinar inmunoglobulinas y Ac antitransglutaminasa tisular 0 antigliadina deaminada.

Los análisis de heces son de especial relevancia, y deben incluir el estudio microscópico para valorar la existencia de leucocitos, sangre o grasa (tinción con Sudán). Además, se solicitarán determinación de parásitos, coprocultivo y toxina de Clostridium difficile si hubo tratamiento antibiótico previo1. Si estuviera justificado un estudio más exhaustivo se puede medir el peso, ph, grasa total (Van de Kamer), iones, gap osmótico que es igual a $290-[2 x(\mathrm{Na}+\mathrm{K})]$. Si el valor es $>125$ se trata de una diarrea osmótica $y<50$, diarrea secretora. Por otra parte, es posible determinar la presencia de algunos laxantes en heces si se sospecha ingesta subrepticia de los mismos.

Un análisis de más reciente adquisición es el de la calprotectina fecal; se trata de una proteína que deriva de los neutrófilos y se excreta en las heces cuando hay inflamación de la mucosa. Por tanto, su utilidad radica en la diferenciación de la diarrea inflamatoria. En la colitis ulcerosa y en la enfermedad de Crohn activas, sus valores están elevados y guardan relación con el grado de inflamación². Cuando la mucosa intestinal está sana, como es el caso del Sll, los resultados son normales?

Otras pruebas complementarias diagnósticas, de suma utilidad e imprescindibles en algunos casos son: la endoscopia digestiva alta y baja con toma de biopsias en duodeno, yeyuno, colon e íleon terminal según proceda, ecoendoscopia (estudio del páncreas y vía biliar), ecografía convencional, TAC, colangiopancreato RMN y entero RMN.

\section{Entidades clínicas más frecuentes que cursan con dia- rrea crónica}

Sindrome de intestino irritable. Según los criterios de Roma III (2006) se caracteriza por dolor ó malestar abdominal, de 6 meses de evolución, que en los últimos 3 meses y al menos durante 3 días al mes, se asocia con mejoría tras la defecación y cambios en la frecuencia deposicional y/o morfología de las heces 5 .

En el SIl no hay afectación del estado general, la diarrea se inicia al despertar, es de predominio matutino o postpandrial y respeta el sueño nocturno. Las heces son blandas o semilíquidas, a veces con moco y nunca con sangre, se producen por hipermotilidad con urgencia deposicional y sensación de evacuación incompleta².

Afecta al $10 \%$ de la población ${ }^{6}$ y la mayoría son formas leves, solo el 5\% es muy sintomático. Predomina en el sexo femenino y suele existir una personalidad ansiosa de base. Empeoran con el estrés, y el tratamiento habitual es con reguladores de la motilidad intestinal y/o psicotropos ${ }^{1}$. Si no se consigue una mejoría con esta medicación, deben de valorarse otras posibilidades diagnósticas como son: intolerancia a la lactosa, enfermedad celiaca, colitis microscópica y malabsorción de sales biliares, las cuales pueden dar un cuadro clínico muy similar al del SII3.

El diagnóstico se hace fundamentalmente por la historia clínica y exclusión de enfermedad orgánica. En este sentido, el screening con calprotectina en heces puede evitar la realización de colonoscopias para descartar organicidad, puesto que, en principio, solamente estarían indicadas en los casos positivos ${ }^{3,7}$.

Cuando la diarrea no se acompaña de dolor o malestar abdominal y el resto de caracteres clínicos es muy similar al S.I.I, hablamos de diarrea funcional ${ }^{8}$.

Diarreas asociadas a la toma de fármacos. Este es un capitulo muy importante en nuestro medio, en donde la politerapia 
está a la orden del día. La diarrea es el efecto colateral más constatado de la mayoría de los medicamentos. La lista es muy grande y se dice que hasta 800 fármacos y productos de herboristería pueden producir diarrea ${ }^{8}$, El mecanismo por el cual se produce es, osmótico, secretor, hipermotilidad 0 mixto ${ }^{8}$. Suele existir una relación temporal causa-efecto pero también puede aparecer de forma diferida durante un tratamiento crónico y sin modificar la posología. Los grupos terapéuticos y fármacos más usados que puede dar diarrea son:

- Inhibidores de la secreción gástrica (IBPS): omeprazol y similares.

- Inhibidores de la HMG-CoA reductasa (estatinas).

- Inhibidores de la recaptación de serotonina (antidepresivos).

- Antihipertensivos (IECAs y ARA-II).

- Antidiabéticos (metformina).

- Antibióticos.

- Agentes quimioterápicos, AINEs, antiarrítmicos etc.

Desde el punto de vista práctico, es conveniente recordar que ante un enfermo que consulta por diarrea prolongada sin deterioro significativo del estado general, es preciso preguntar siempre por los fármacos que está tomando y considerar este diagnóstico como primera posibilidad.

Diarrea infecciosa. La diarrea crónica de causa infecciosa es poco frecuente en el mundo occidental. En este contexto, los gérmenes más habituales son: yersinia, aeromona, shigella, salmonella y campylobacter ${ }^{3,9}$. La toma de antibióticos en los 3 meses previos a la aparición de la diarrea, puede facilitar su aparición por cambios en la flora intestinal, y por otra parte por crecimiento de clostridium difficile y producción de toxinas ${ }^{9}$.

Las infecciones por virus $u$ hongos son infrecuentes en el individuo inmunocompetente. Las infecciones por parásitos no son habituales en nuestro entorno y pueden verse en individuos que viajaron a áreas endémicas. Las giardias y amebas son los parásitos más habituales, que se transmiten por aguas contaminadas y el estudio en heces de las mismas sirve para el diagnóstico, aunque en ocasiones son necesarios estudios serológicos.

En este apartado de diarreas crónicas de causa infecciosa hay dos enfermedades poco frecuentes pero de gran relevancia clínica que merecen mención especial; éstas son la tuberculosis intestinal y la enfermedad de Whipple.

Tuberculosis (TBC) intestinal: representa el 3'5\% de los casos de TBC extrapulmonar y coexiste con enfermedad pulmonar activa o inactiva el $75 \%$ de los $\operatorname{casos}^{10}$. Se presenta con síntomas inespecíficos, dolor abdominal, pérdida de peso, diarrea, sangre en heces, fiebre o febrícula9 ${ }^{9}$ Puede afectar a cualquier parte del tubo digestivo, si bien es más frecuente a nivel de íleon terminal y ciego. Las imágenes endoscópicas e histológicas pue- den simular la enfermedad de Crohn y es necesario un alto índice de sospecha para su diagnóstico, que puede confirmarse en las muestras de heces y sobre todo en una biopsia, ya sea con cultivo en medio de Lowenstein (poco sensible) o bien con técnicas de PCR, que son más sensibles y específicas (> 85\%) y añaden la ventaja de la rapidez en los resultados (24-48 h) ${ }^{10}$.

Enfermedad de Whipple: es muy poco frecuente pero con gran expresividad clínica. Esta producida por un bacilo gram positivo, Tropheryma Whippelli, que penetra en el organismo por vía digestiva, produce afectación de intestino delgado con diarrea y síndrome de malabsorción muy florido (sobre todo en las formas clásicas); se disemina por el sistema linforeticular y puede afectar también al sistema nervioso central.

Además del síndrome de malabsorción, puede producir, artralgias, artritis, fiebre, adenopatías y síntomas neurológicos con predominio de deterioro cognitivo.

El diagnóstico se realiza habitualmente por endoscopia y biopsia duodenal que demuestra la existencia de macrófagos cargados de material PAS positivo. En algunas ocasiones pueden ser necesarias técnicas de inmunohistoquímica o PCR en muestras histológicas o fluidos biológicos.

Colitis microscópica es una causa frecuente de diarrea crónica, afecta principalmente a adultos de 60-70 años y predomina el sexo femenino ${ }^{11}$. La incidencia ha aumentado en los últimos años y se aproxima a la de la ElCl, 4-6/100.000 habitantes ${ }^{11,12}$. Se caracteriza clínicamente por diarrea acuosa que no respeta el sueño, dolorimiento abdominal, pérdida de peso y en ocasiones incontinencia fecal. Su etiología es desconocida, aunque hay series que la asocian a la toma de fármacos como son AINES e inhibidores de la recaptación de serotonina entre otros ${ }^{13}$.

Macroscópicamente la mucosa colónica es normal, y el diagnóstico se hace por biopsia del colon proximal; la afectación puede ser parcheada por lo que deben obtenerse varias muestras de colon derecho y transverso ${ }^{3}$. Según los hallazgos histológicos se clasifica en colitis linfocítica o colágena.

No es infrecuente su asociación con enfermedades autoinmunes como la enfermedad celíaca, artritis reumatoide, enfermedades tiroideas, asma y diabetes tipo $\mathrm{I}^{11}$. El tratamiento con budesonida oral mejora la sintomatología y el pronóstico es bueno en un plazo 2-3 años ${ }^{11}$. Las formas leves pueden tratarse sintomáticamente.

Enfermedad celiaca: es relativamente frecuente y de presentación clínica muy variable; las formas clásicas que cursan con diarrea y síndrome de malabsorción evidente son inusuales.

Como prueba de screening deben realizarse los anticuerpos antitransglutaminasa tisular $\lg \mathrm{A} 0$ antigliadina deaminada, 
Tabla 2. Causas de diarrea crónica

\begin{tabular}{ll}
\hline Acuosa & Fármacos \\
& Síndrome de Intestino Irritable \\
& Colitis microscópica \\
& Malabsorción de carbohidratos \\
& Ingesta de laxantes \\
& Malabsorción de sales biliares \\
& Neoplasias: Adenocarcinoma colorrectal, Adenoma \\
& velloso, Linfoma \\
& Parásitos \\
& Tumores neuroendocrinos \\
& Diarrea facticia \\
& Pancreatitis crónica \\
& Sobrecrecimiento bacteriano \\
& Enfermedad celiaca \\
& Enfermedad de Crohn \\
& Linfoma intestinal \\
& Postquirúrgica: Intestino corto, Colecistectomía, \\
& Bypass, Vagotomía, Asas ciegas \\
& Postradiación \\
& Enfermedad de Whipple \\
& Enteritis eosinofilica \\
& Amiloidosis \\
\hline Grasa & ElCl (colitis ulcerosa, Crohn) \\
Infecciones & Bacterias: Cl. difficile, Salmonella, Shigella, Yersinia, \\
& Campylobacter, TBC \\
& Fármacositos: Amebiasis, Giardia \\
& Neoplasiasión \\
\hline Inflamatoria & \\
\hline &
\end{tabular}

con alta sensibilidad y especificidad' ${ }^{1}$. La confirmación diagnóstica se hace por biopsia duodenal, y en caso de duda o para completar el estudio, mediante la determinación de marcadores genéticos HLA, DQ2 y DQ8, los cuales están casi siempre presentes en la enfermedad, y cuya negatividad va en contra de enfermedad celiaca ${ }^{14}$.

En este número de la revista se presenta un caso clínico de celiaca del adulto en donde se comentan los aspectos más relevantes de la enfermedad.

Diarrea acuosa: ya comentada en al apartado previo de fisiopatología, es la forma clínica más frecuente de presentación de una diarrea crónica. Las causas son muy variadas y una vez más la historia clínica es fundamental para orientar el diagnóstico. Lo primero a preguntar es sobre la ingesta de fármacos y otros productos; valorar la tolerancia y malabsorción de hidratos de carbono como la lactosa ${ }^{15}$, fructosa y sorbitol. Es preciso recordar que también producen diarrea acuosa el SII, la colitis microscópica, la enfermedad celiaca y las infecciones, tal como se expuso anteriormente ${ }^{1}$. También hay que tener presente la posibilidad de malabsorción de sales biliares especialmente si hay afectación del íleon terminal, resección ileal ó diarrea post-colecistectomía.

El prototipo de diarrea acuosa secretora es la producida por tumores neuroendocrinos, muy poco frecuentes, donde en caso de sospecha clínica se pedirán las hormonas correspondientes (gastrina, serotonina, calcitonina, VIP, etc). El volumen de secreción es alto y el gap osmótico <50. En este número de la revista se publica un caso de vipoma y los autores hacen una revisión exhaustiva de este tipo de diarreas.

A modo de resumen, el manejo de una diarrea crónica es variable debido al gran número de etiologías (tabla 2) y no es fácil hacer un esquema práctico aplicable en todos los casos. El uso racional de los datos de la historia clínica, examen físico y pruebas complementarias seleccionadas convenientemente en cada paciente permite hacer un diagnóstico correcto en la mayoría de los enfermos. Conviene no olvidar que en la práctica clínica diaria, es más frecuente una enfermedad habitual con presentación atípica que una enfermedad rara; lo raro siempre es raro.

\section{Bibliografía}

1. Sandhu DK, Surawicz C. Update on chronic diarrhea: a run-through for the clinician. Curr Gastroenterol Rep. 2012; 14:421-7.

2. Bonis PAL, LaMont JT. Approach to the adult with chronic diarrhea in developed countries. In: UpToDate, Basow, DS (Ed), UpToDate, Waltham, MA, 2013.

3. Juckett G, Trivedi R. Evaluation of chronic diarrhea. Am Fam Physician. 2011 15; 84:1119-26.

4. Headstrom PD, Surawicz CM. Chronic diarrhea. Clin Gastroenterol Hepatol. 2005; 3:734-7.

5. Longstreth GF, Thompson WG, Chey WD, Houghton LA, Mearin F, Spiller RC. Functional bowel disorders. Gastroenterology. 2006; 130:1480-91.

6. Mearin F, Badía X, Balboa A, Baró E, Caldwell E, Cucala M, Díaz-Rubio M, Fueyo A, Ponce J, Roset M, Talley NJ. Irritable bowel syndrome prevalence varies enormously depending on the employed diagnostic criteria: comparison of Rome II versus previous criteria in a general population. Scand J Gastroenterol. 2001; 36:1155-61.

7. Mindemark M, Larsson A. Ruling out IBD: estimation of the possible economic effects of pre-endoscopic screening with F-calprotectin. Clin Biochem. 2012; 45:552-5.

8. Abraham BP, Sellin JH. Drug-induced, factitious, \& idiopathic diarrhoea. Best Pract Res Clin Gastroenterol. 2012; 26:633-48.

9. Kaiser L, Surawicz CM. Infectious causes of chronic diarrhea. Best Pract Res Clin Gastroenterol. 2012; 26:563-71.

10. García-Castro JM, Javier-Martínez R, López-Gómez M, Hidalgo-Tenorio C, López-Ruz MÁ, Jiménez-Alonso J. Tuberculosis intestinal que simula carcinoma colorrectal diseminado. Gastroenterología y Hepatología. 2013; 36:461-3.

11. Nyhlin N, Bohr J, Eriksson S, Tysk C. Microscopic colitis: a common and an easily overlooked cause of chronic diarrhoea. Eur J Intern Med. 2008; 19:181-6.

12. Yen EF, Pardi DS. Non-IBD colitides (eosinophilic, microscopic). Best Pract Res Clin Gastroenterol. 2012; 26:611-22.

13. Fernández-Bañares F, Esteve M, Espinós JC, Rosinach $M$, Forné $M$, Salas $A$, Viver JM. Drug consumption and the risk of microscopic colitis. Am J Gastroenterol. 2007; 102:324-30

14. Kaukinen K, Partanen J, Maki M, Collin P. HLA-DQ typing in the diagnosis of celiac disease. Am J Gastroenterol 2002; 97:695-9.

15. Montgomery RK, Grand RJ, Büller HA. Lactose intolerance. In: UpToDate, Basow, DS (Ed), UpToDate, Waltham, MA, 2013. 